\section{Insegurança alimentar em domicílios brasileiros com crianças menores de cinco anos}

\author{
Food insecurity in Brazilian families with children \\ under five years of age
}

\section{Inseguridad alimentaria en los hogares brasileños con niños menores de cinco años}

Ana Paula Poblacion 1 Leticia Marín-León 2 Ana Maria Segall-Corrêa 2 Jonas Augusto Silveira 1 José Augusto de Aguiar Carrazedo Taddei 1
${ }^{1}$ Universidade Federal de São Paulo, São Paulo, Brasil.

2 Faculdade de Ciências Médicas, Universidade Estadual de Campinas Campinas, Brasil.

Correspondência A. P. Poblacion Disciplina de Nutrologia, Departamento de Pediatria Universidade Federal de São Paulo.

Rua Loefgren 1647, São Paulo, SP 04040-032, Brasil. anapoblacion@yahoo.com.br

\begin{abstract}
This article analyzes food insecurity and hunger in Brazilian families with children under five years of age. This was a nationally representative cross-sectional study using data from the National Demographic and Health Survey on Women and Children (PNDS-2006), in which the outcome variable was moderate to severe food insecurity, measured by the Brazilian Food Insecurity Scale (EBIA). Prevalence estimates and prevalence ratios were generated with $95 \%$ confidence intervals. The results showed a high prevalence of moderate to severe food insecurity, concentrated in the North and Northeast regions (30.7\%), in economic classes D and E (34\%), and in beneficiaries of conditional cash transfer programs (36.5\%). Multivariate analysis showed that the socioeconomic relative risks (beneficiaries of conditional cash transfers), regional relative risks (North and Northeast regions), and economic relative risks (classes $D$ and $E$ ) were 1.8, 2.0 and 2.4, respectively. Aggregation of the three risks showed $48 \%$ of families with moderate to severe food insecurity, meaning that adults and children were going hungry during the three months preceding the survey.
\end{abstract}

Food Security; Social Inequity; Social Vulnerability; Health Surveys; Hunger

\section{Resumo}

Este artigo se propõe a estudar o processo de insegurança alimentar e fome em domicílios brasileiros com crianças menores de cinco anos. $E$ um estudo transversal com representatividade nacional executado com dados da Pesquisa Nacional de Demografia e Saúde da Criança e da Mulher (PNDS-2006) tendo como variável dependente a insegurança alimentar moderada e grave $(I A M+G)$, medida através da Escala Brasileira de Insegurança Alimentar (EBIA). Foram geradas estimativas de prevalência e razão de prevalência com intervalos de $95 \%$ de confiança. Os resultados apontam para alta prevalência de IAM+G concentrada nas regiões Norte e Nordeste (30,7\%), nas classes econômicas De E (34\%) e em beneficiários de Programas de Transferência de Renda (PTR; 36,5\%). O modelo de análise multivariada constatou que os riscos sociais (beneficiário de PTR), regionais (Norte e Nordeste) $e$ econômicos (classes D e E) eram de 1,8, 2,0 e 2,4, respectivamente. Agregando-se os três riscos observou-se que $48 \%$ dos domicílios encontravamse em IAM+G, ou seja, crianças e adultos passaram fome nos três meses anteriores ao inquérito.

Segurança Alimentar e Nutricional; Iniquidade Social; Vulnerabilidade Social; Inquéritos Epidemiológicos; Fome 


\section{Introdução}

O Brasil é uma das sociedades mais desiguais do planeta. Embora esteja colocado entre as seis maiores economias mundiais, quando se considera o Índice de Desenvolvimento Humano (IDH) o Brasil ocupa a 84a posição 1, quando se considera o Índice de Gini o país apresenta o terceiro pior índice de desigualdade de renda 2 . Em adição, dentro do território nacional também são observadas disparidades regionais, sendo as macrorregiões Centro-oeste, Sudeste e Sul aquelas com índices de desenvolvimento maior, e Norte e Nordeste, as menos desenvolvidas 3.

Estabelecer novos indicadores de avaliação da desigualdade tem sido objeto de estudo de diversas áreas do conhecimento. Durante os anos 90, o Departamento Americano de Agricultura (USDA) liderou os esforços para o desenvolvimento de uma escala capaz de medir diretamente a insegurança alimentar domiciliar a partir das escalas de Radimer/Cornell 4,5 e do Projeto Comunitário de Identificação de Fome Infantil (CCHIP) 6, denominada U.S. Household Food Security Survey Measure (HFSSM) 7,8.

Diversos países traduziram e validaram a HFSSM 9. Em 2004, o Brasil empregou a metodologia deste instrumento e validou sua própria versão nomeada de Escala Brasileira de Insegurança Alimentar (EBIA), com o propósito de medir diretamente a percepção da insegurança alimentar e da fome em nível domiciliar relacionado com a dificuldade de acesso regular e permanente a alimentos de qualidade e em quantidade suficiente; e, a partir da evolução dos perfis de insegurança avaliar o efeito dos programas e das políticas públicas em nível populacional 8,10.

Vários estudos realizados no país demonstram que a EBIA é adequada para a identificação de grupos ou populações de risco em nível local, regional ou nacional, e para o estudo dos determinantes e das consequências da insegurança alimentar 11 .

Em 2006, a Pesquisa Nacional de Demografia e Saúde da Criança e da Mulher (PNDS 2006) utilizou a EBIA para coletar dados de (in)segurança alimentar nos domicílios com mulheres em idade fértil e, se houvesse, pelo menos um morador menor de 18 anos de idade. Dessa forma foi possível gerar informações referentes aos indivíduos em processo de crescimento, portanto, em potencial risco nutricional. Dentre estes, crianças nos primeiros cinco anos vivenciam mudanças marcantes nos hábitos alimentares e no desenvolvimento cognitivo, linguístico, social e emocional, caracterizando-se como de maior vulnerabilidade biológica. Por isso, é prioritário garantir a esse grupo etário o acesso regular e perma- nente à alimentação, pois experimentar restrição alimentar quantitativa importante, ou episódios que configuram situação de fome durante este período, reflete negativamente na sobrevivência, no crescimento físico e no desenvolvimento neuropsicomotor dos futuros cidadãos do país.

Sendo assim, esta pesquisa se propõe a estudar o processo da insegurança alimentar e da fome no país, a partir dos dados da amostra de domicílios com residentes menores de cinco anos de idade, coletados pela PNDS 2006, e ainda parcialmente explorados, visando gerar conhecimento específico para o planejamento e a implementação de políticas públicas mais efetivas a fim de promover melhores condições de nutrição e saúde entre lactentes e pré-escolares.

\section{Metodologia}

\section{Desenho do estudo}

O presente estudo utilizou dados de domínio público da terceira edição da PNDS 2006, que estão em consonância com a 5a fase do Projeto Measure DHS. Financiada pelo Ministério da Saúde, a PNDS foi coordenada pelo Centro Brasileiro de Análise e Planejamento (CEBRAP) que utilizou o Instituto Brasileiro de Opinião Pública e Estatística (IBOPE) para a execução do trabalho de campo. A PNDS é uma pesquisa domiciliar por amostragem probabilística complexa em dois estágios, sendo o primeiro estágio as áreas censitárias e o segundo os domicílios. Teve como objetivo descrever o perfil individual e domiciliar da população feminina em idade fértil e das crianças menores de cinco anos. Concomitantemente, coletou dados sobre a (in)segurança alimentar no domicílio utilizando a EBIA. A metodologia da pesquisa, incluindo a seleção amostral, os procedimentos de coleta de dados, a consistência dos dados, as técnicas de expansão para amostras complexas e aspectos éticos, estão descritos em publicação específica 12 .

\section{Variável dependente}

A (in)segurança alimentar foi medida por um instrumento adaptado e validado para a população brasileira intitulado EBIA, sendo que as perguntas referem-se aos três meses antecedentes à pesquisa, e o número de respostas afirmativas determina a classificação em: segurança alimentar (0); insegurança alimentar leve (1-5); insegurança alimentar moderada (6-10); insegurança alimentar grave (11-15). No domicílio em segurança alimentar todas as pessoas têm acesso regular e permanente a alimentos de qualidade 
e em quantidade suficiente. A insegurança alimentar leve (IAL) identifica o comprometimento da qualidade da alimentação em detrimento da manutenção da quantidade percebida como adequada para os moradores do domicílio. A insegurança alimentar moderada (IAM) remete à insuficiência de alimentos com restrições de sua quantidade ou mudança nos padrões usuais da alimentação entre os adultos. Os domicílios que apresentam insegurança alimentar grave (IAG) são caracterizados pela quebra do padrão usual da alimentação com comprometimento da qualidade e redução da quantidade de alimentos nas refeições de todos os membros da família, inclusive das crianças residentes nesse domicílio, podendo ainda incluir a experiência de fome $8,11,13,14$.

Quando houvesse resposta "não sabe" às perguntas relativas à condição de acesso aos alimentos (1a a 4a questão), a entrevista era interrompida a fim de evitar rotular como inseguro, um domicílio com alta probabilidade de segurança alimentar. Para a análise, 17 domicílios foram excluídos.

Para fins metodológicos, a PNDS 2006 utilizou a EBIA modificada, contendo 16 perguntas, sendo a questão número cinco desdobrada em duas. Para a análise, as duas questões foram reagrupadas considerando "não" quando ambas tivessem respostas negativas ou "não sabe" para uma pergunta e "não" para outra. Para qualquer outra possibilidade a resposta foi marcada como "sim".

Para estimativas de prevalências utilizou-se esta variável em seus quatro níveis, sendo segurança alimentar, IAL, IAM, IAG. Para os modelos bi e multivariado utilizou-se a variável dicotomizada, sendo considerados em risco os domicílios em IAM ou IAG (“IAM+G") e sem risco quando estes domicílios estavam em segurança alimentar ou IAL (“SA+IAL”).

\section{Variáveis independentes}

\section{a) Macrorregião administrativa}

As estimativas de prevalências foram calculadas para as cinco macrorregiões administrativas Norte (N), Nordeste (NE), Centro-oeste (CO), Sudeste (SE) e Sul (S). Já para as análises bi e multivariadas a variável foi dicotomizada, sendo o Brasil "mais desenvolvido" composto por $\mathrm{CO}+\mathrm{SE}+\mathrm{S}$ e o Brasil “menos desenvolvido" por N+NE.

\section{b) Situação de domicílio}

Para gerar estimativas de prevalências segundo a (in)segurança alimentar, considerou-se a situação do domicílio, urbano ou rural.

\section{c) Classificação econômica}

A renda per capita ou familiar, apesar de medir diretamente o valor econômico disponível para compra de bens, serviços e alimentos, é uma variável instável, por isso o poder de compra pode ser utilizado como uma medida proxy da renda. Para avaliar a condição econômica utilizouse o Critério de Classificação Econômica Brasil (CCEB), que quantifica oito bens duráveis do domicílio e o número de empregados mensalistas, além do grau de instrução do chefe da família, gerando pontos de corte para a determinação do poder aquisitivo da pessoa ou da família. As variáveis que compõem o critério foram coletadas pela PNDS 2006, e de acordo com os intervalos de pontuação da escala definiram-se oito classes econômicas de Al a E (Associação Brasileira de Estudos Populacionais. http://www.abep.org/ novo/content.aspx?ContentID=139). Para prevalências, análises bi e multivariadas, foi utilizada a variável dicotomizada, agrupando as classes "mais abastadas" de A1 a C2, e as classes "menos abastadas" D+E.

\section{d) Programas de transferência de renda}

Para compor esta variável, considerou-se "sim" quando havia o recebimento monetário de pelo menos um programa no domicílio: Bolsa Família, Bolsa-Escola Municipal, Benefício de Prestação Continuada (BPC), Auxílio-gás, Cartão Alimentação, Programa de Erradicação do Trabalho Infantil (PETI), Agente Jovem. Em todas as estatísticas utilizou-se a variável dicotômica, sendo considerada situação de risco quando algum morador do domicílio "recebe PTR".

\section{Elegibilidade e critério de seleção}

Dos 27.477 filhos das mulheres elegíveis da PNDS 2006, foram selecionados os menores de cinco anos de idade na data da entrevista, residentes com suas mães e que dispunham da EBIA preenchida corretamente, totalizando 4.800 crianças.

Como a unidade de estudo é o domicílio, foi necessário considerar somente uma criança menor de cinco anos por residência, para que a representação daquela unidade amostral não viesse a ser duplicada na análise dos dados. Sendo assim, quando uma mesma mulher da amostra tinha mais do que um filho menor de cinco anos, foi considerado somente o de maior idade ( $\mathrm{n}=784$ ). Quando havia duas ou mais crianças menores de cinco anos, filhos de mães diferentes, residentes no mesmo domicílio, foram excluídas as crianças mais novas $(n=61)$. Procedeu-se desta forma no intuito de identificar a criança menor 
de cinco anos que mais tempo havia vivido na condição de (in)segurança alimentar.

Com o mesmo objetivo, nas situações em que existiam gemelares, selecionou-se o gêmeo que havia nascido em melhores condições. No primeiro passo considerou-se apenas o de maior peso ao nascimento anotado no cartão da criança. Caso não existisse essa informação foi selecionado o de maior peso ao nascer referido pela mãe. Por fim, caso não houvesse ambas as informações considerou-se apenas o primeiro listado no número de ordem do filho nascido vivo $(\mathrm{n}=35)$. Após as exclusões, 3.920 domicílios foram considerados para análise, representando 11.779 .686 domicílios quando a amostra é expandida.

\section{Análise dos dados}

Para gerenciar e analisar os bancos de dados utilizou-se o pacote estatístico Stata/IC 12 (Stata Corp., College Station, Estados Unidos). A fim de considerar os procedimentos de amostragem e evitar que as estimativas obtidas sejam viciadas ou incorretas, o efeito do plano amostral foi considerado nas análises ${ }^{15}$. Na PNDS, a escolha do plano amostral utilizado depende da origem e do cruzamento das variáveis contidas nos diferentes bancos de dados, oriundos de questionários diferentes: o domiciliar e o da mulher. Como o tamanho amostral utilizado nesta pesquisa é oriundo do banco PNDS2006_BR_FILHOS.dta, foi utilizado o EPA da mulher 16 .

Os parâmetros e os respectivos intervalos de $95 \%$ de confiança foram estimados com o peso amostral que considera o efeito do desenho amostral. Já na variável PTR empregou-se somente o desenho amostral. Nas análises descritivas foi utilizado o teste qui-quadrado com correção de segunda ordem de Rao-Scott 17, considerando significante quando $\mathrm{p}<0,05$. A Regressão de Poisson 18 foi utilizada nas análises bi e multivariada considerando as variáveis com $\mathrm{p}<0,20$ na bivariada elegíveis para inclusão na multivariada. Permaneceram no modelo final as variáveis com $\mathrm{p}<0,05$.

Os riscos agregados foram calculados com as estimativas de proporções para as categorias identificadas pelos valores de cada uma das variáveis, identificando prevalências das subamostras que apresentavam um, dois ou os três condições de risco. Posteriormente, considerando a expansão da amostra, foram calculados tanto a proporção quanto o número estimado de domicílios com crianças menores de cinco anos que viviam em $\mathrm{IAM}+\mathrm{G}$.

\section{Aspectos éticos}

Os dados da PNDS foram coletados segundo os critérios estabelecidos pela Declaração de Helsinque, tendo todos os procedimentos envolvendo seres humanos aprovados pelo Comitê de Ética do Centro de Referência e Treinamento em DST/ AIDS do Secretaria de Estado da Saúde de São Paulo. Em adição, este trabalho foi aprovado pelo Comitê de Ética em Pesquisa da Universidade Federal de São Paulo/Hospital São Paulo, sob o no $2.117 / 11$.

\section{Resultados}

A Tabela 1 apresenta informações que demonstram tanto as altas prevalências de insegurança alimentar leve, moderada e grave nos domicílios com crianças menores de cinco anos no país como também as grandes disparidades macrorregionais e urbano-rural. São ainda apontadas as variações dos graus de insegurança alimentar nas diferentes categorias de condição econômica e vínculo ao PTR.

Considerando que a $\mathrm{IAM}+\mathrm{G}$ é uma situação familiar de privação de alimentos, registra-se que 17,5\% dos domicílios com crianças menores de cinco anos encontravam-se nesta situação no Brasil de 2006. Os desníveis macrorregionais da condição de $\mathrm{IAM}+\mathrm{G}$ podem ser ilustrados pelos contrastes entre as regiões Norte e Sul com 34,5\% e 7,8\%, respectivamente. Embora não tão marcantes quanto as diferenças macrorregionais, também são bastante desiguais as condições de $I A M+G$ entre a região rural $(23,5 \%)$ e urbana $(16,2 \%)$. A Tabela 1 revela ainda a estreita relação das classes D+E, identificadas pela menor capacidade de consumo, com alta proporção de $\mathrm{IAM}+\mathrm{G}(34,0 \%)$. Evidencia-se também que viviam em situação de $\mathrm{IAM}+\mathrm{G}$ mais de um terço (36,5\%) das famílias com crianças menores de cinco anos que participavam dos PTR em 2006.

De forma consistente são evidenciados tanto nas análises bivariadas quanto no modelo de análise multivariada apresentados na Tabela 2 que estar domiciliado nas macrorregiões Norte ou Nordeste (RP = 2,0; IC95\%: 1,7; 2,3), pertencer às classes econômicas $\mathrm{D}$ ou $\mathrm{E}(\mathrm{RP}=2,4$; IC95\%: $2,1 ; 2,8)$ e participar de PTR (RP = 1,8; IC95\%: $1,6 ; 2,0)$ associam-se independentemente com $\mathrm{IAM}+\mathrm{G}$.

A Figura 1 mostra que $17,5 \%$ das residências brasileiras com moradores menores de cinco anos estavam em IAM+G nos três meses que antecederam a entrevista, representando mais de dois milhões de domicílios após expandir a amostra. Quando são consideradas as localizadas 
Tabela 1

Distribuição da (in)segurança alimentar em domićilios com crianças menores de cinco anos, segundo macrorregião, situação do domicílio, critério de classificação econômica da Associação Brasileira de Estudos Populacionais (CCEB/ABEP) e programas de transferência de renda. Pesquisa Nacional de Demografia e Saúde, Brasil, 2006.

\begin{tabular}{|c|c|c|c|c|c|c|}
\hline \multirow[t]{2}{*}{ Características } & \multirow{2}{*}{$\begin{array}{l}\text { Domicílios } \\
\text { [n (\%)] }\end{array}$} & \multirow{2}{*}{$\begin{array}{c}\text { Segurança } \\
\text { alimentar } \\
\text { [\% (IC95\%)] }\end{array}$} & \multicolumn{3}{|c|}{ Insegurança alimentar [\% (IC95\%)] } & \multirow[t]{2}{*}{ Valor de $p$ * } \\
\hline & & & Leve & Moderada & Grave & \\
\hline Brasil e macrorregiões & & & & & & 0,0000 \\
\hline Brasil & $3.920(100,0)$ & $54,5(50,9 ; 58,1)$ & $27,9(25,3 ; 30,7)$ & $12,3(10,5 ; 14,5)$ & $5,2(4,3 ; 6,3)$ & \\
\hline Norte & $774(19,7)$ & $39,2(34,0 ; 44,5)$ & $26,4(22,3 ; 30,9)$ & $19,8(16,3 ; 23,8)$ & $14,7(11,6 ; 18,4)$ & \\
\hline Nordeste & $740(18,9)$ & $40,5(34,9 ; 46,4)$ & $30,1(25,1 ; 35,6)$ & $21,0(17,8 ; 24,7)$ & $8,4(6,2 ; 11,3)$ & \\
\hline Centro-oeste & $817(20,8)$ & $58,6(53,1 ; 63,8)$ & $29,0(24,5 ; 34,0)$ & $9,7(7,4 ; 12,8)$ & $2,6(1,5 ; 4,5)$ & \\
\hline Sudeste & $800(20,4)$ & $61,7(54,4 ; 68,4)$ & $27,9(23,1 ; 33,3)$ & $8,0(5,0 ; 12,6)$ & $2,4(1,5 ; 3,9)$ & \\
\hline Sul & $789(20,1)$ & $68,3(63,6 ; 72,7)$ & $23,8(19,9 ; 28,2)$ & $5,0(3,5 ; 7,2)$ & $2,8(1,5 ; 5,2)$ & \\
\hline Situação do domicílio & & & & & & 0,0407 \\
\hline Urbano & $2.601(66,3)$ & $55,3(51,0 ; 59,4)$ & $28,5(25,6 ; 31,6)$ & $11,3(9,3 ; 13,7)$ & $4,9(3,9 ; 6,0)$ & \\
\hline Rural & $1.319(33,7)$ & $51,3(45,5 ; 57,1)$ & $25,1(20,0 ; 31,1)$ & $16,9(13,5 ; 20,9)$ & $6,6(4,5 ; 9,8)$ & \\
\hline CCEB/ABEP ** & & & & & & 0,0000 \\
\hline A1 a C2 & $2.463(62,8)$ & $66,0(62,1 ; 69,8)$ & $24,5(21,5 ; 27,8)$ & $6,9(5,5 ; 8,7)$ & $2,4(1,7 ; 3,4)$ & \\
\hline$D+E$ & $1.456(37,1)$ & $31,4(26,7 ; 36,6)$ & $34,6(30,1 ; 39,3)$ & $23,2(19,3 ; 27,6)$ & $10,8(8,7 ; 13,2)$ & \\
\hline Programas de transferência de renda *** & & & & & & 0,0000 \\
\hline Pertencer & $1.203(30,7)$ & $34,0(31,1 ; 37,0)$ & $29,5(26,8 ; 32,3)$ & $22,0(19,7 ; 24,5)$ & $14,5(12,3 ; 16,9)$ & \\
\hline Não pertencer & $2.714(69,3)$ & $59,3(57,2 ; 61,4)$ & $27,0(25,3 ; 28,9)$ & $8,9(7,9 ; 10,1)$ & $4,7(3,9 ; 5,7)$ & \\
\hline
\end{tabular}

IC95\%: intervalo de 95\% de confiança.

* Teste qui-quadrado com correção de Rao-Scott;

** 1 entrevista sem informação;

*** 4 entrevistas sem informação.

Tabela 2

Razões de prevalências (RP) de insegurança alimentar moderada + grave (IAM+G) em domicílios com crianças menores de cinco anos para variáveis socioeconômicas e demográficas, estimadas mediante modelo de regressão de Poisson. Pesquisa Nacional de Demografia e Saúde, Brasil, 2006.

\begin{tabular}{|c|c|c|c|c|c|c|c|}
\hline \multirow[t]{2}{*}{ Variáveis/referência } & \multirow[t]{2}{*}{$n / t$} & \multicolumn{3}{|c|}{ Análise bivariada } & \multicolumn{3}{|c|}{ Análise multivariada * } \\
\hline & & RP & $\mathrm{IC} 95 \%$ & Valor de p & RP & IC95\% & Valor de $\mathrm{p}$ \\
\hline \multicolumn{8}{|l|}{ Macrorregião } \\
\hline Norte + Nordeste & $517 / 812$ & 2,8 & 2,$4 ; 3,2$ & 0,000 & 2,0 & 1,$7 ; 2,3$ & 0,000 \\
\hline \multicolumn{8}{|l|}{ CCEB/ABEP } \\
\hline$D+E$ & $545 / 812$ & 3,4 & 3,$0 ; 3,9$ & 0,000 & 2,4 & 2,$1 ; 2,8$ & 0,000 \\
\hline \multicolumn{8}{|c|}{ Programas de Transferência de renda } \\
\hline Pertencer & $439 / 810$ & 2,7 & 2,$3 ; 3,0$ & 0,000 & 1,8 & 1,$6 ; 2,0$ & 0,0000 \\
\hline \multicolumn{8}{|l|}{ Situação de domicílio } \\
\hline Rural & $316 / 812$ & 1,3 & 1,$1 ; 1,5$ & 0,008 & & & \\
\hline
\end{tabular}

CCEB/ABEP: critério de classificação econômica da Associação Brasileira de Estudos Populacionais; IC95\%: intervalo de 95\% de confiança; n/t: número de domicílios na categoria de risco/amostra total de domicílios com residentes menores de cinco anos.

* Controlado por situação de domicílio. 
Prevalências (\%) de insegurança alimentar moderada + grave (IAM+G) em domicílios com crianças menores de cinco anos, para subgrupos de riscos agregados. Pesquisa Nacional de Demografia e Saúde, Brasil, 2006.

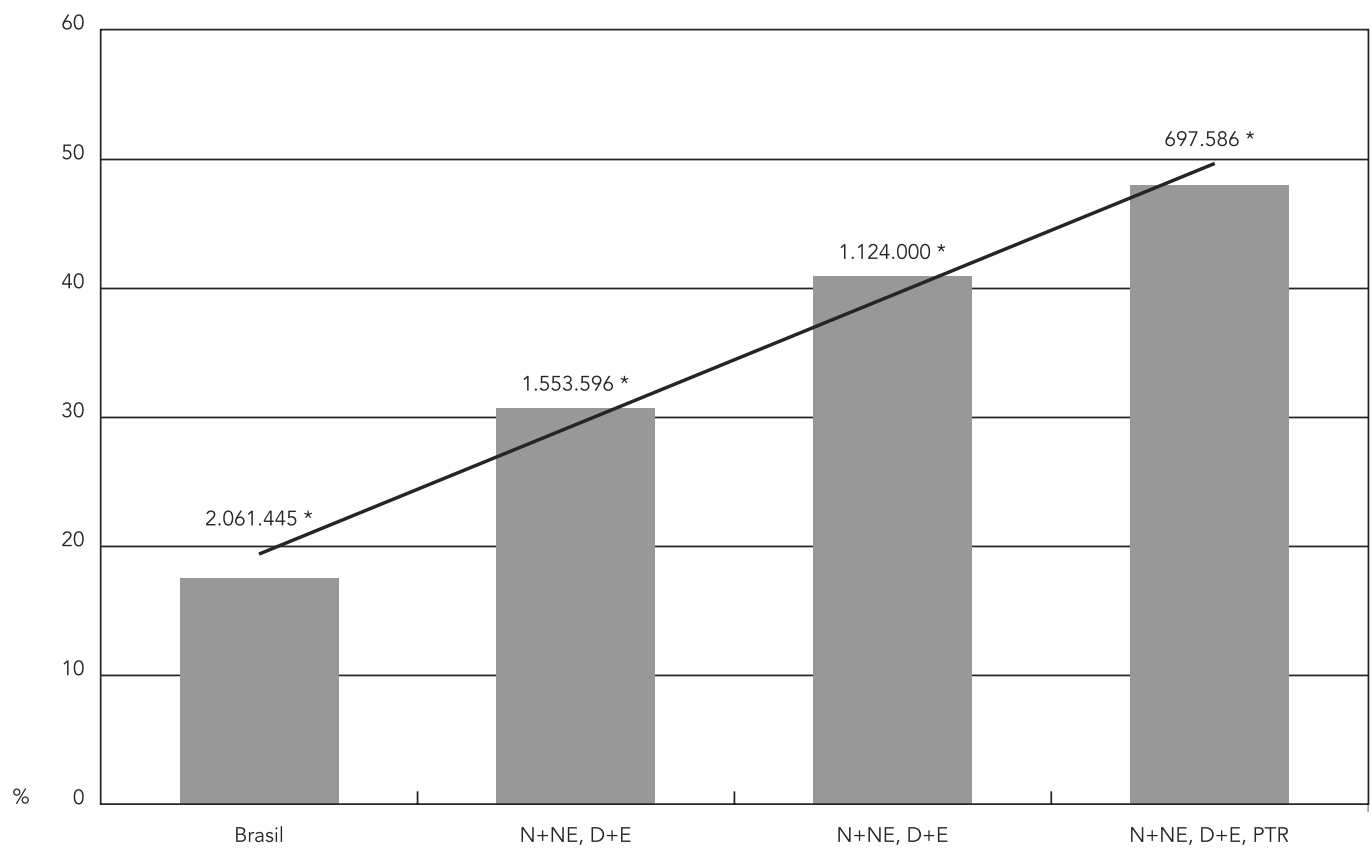

$\mathrm{N}+\mathrm{NE}$ : residir nas macrorregiões Norte e Nordeste; $\mathrm{D}+\mathrm{E}$ : pertencer às classes econômicas D e E (CCEB/ABEP);

PTR: pertencer a programas de transferência de renda.

* Número de domicílios com aplicação de fator de expansão amostral.

nas regiões Norte ou Nordeste, a prevalência aumentava para $30,7 \%$, o equivalente a mais de um milhão e meio de domicílios após expansão da amostra. Quando, além dessa situação, o domicílio apresentasse outros dois fatores de risco, pertencer às classes econômicas $\mathrm{D}$ ou $\mathrm{E}$ e pertencer a PTR, a prevalência passava a ser $48 \%$ (cerca de setecentos mil domicílios segundo a amostra expandida). Ou seja, os riscos agregados identificam grupos com características nos quais quase que triplica a proporção de domicílios com adultos e crianças menores de cinco anos passando fome em 2006.

\section{Discussão}

Desagregar informações sobre a situação de insegurança alimentar por subgrupo da população dá visibilidade para condições de vulnerabilidade que são relevantes ao planejamento ou reformulação de políticas públicas. Para tanto, é im- portante a escolha do delineamento de pesquisa uma vez que o delineamento transversal pode limitar a capacidade de inferência causal, pois não prova a existência de uma sequência temporal. Por outro lado, este delineamento permite obter prevalências e determinar associações, medidas epidemiológicas de grande utilidade na gestão e programação em Saúde Pública. No entanto, apenas estudos longitudinais, ainda não disponíveis no Brasil, poderiam sanar as limitações metodológicas referidas.

Tradicionalmente a segurança alimentar e nutricional vinha sendo monitorada por indicadores indiretos como os socioeconômicos, de disponibilidade de alimentos, de consumo alimentar e os antropométricos; cada qual aferindo um espectro específico sem precisamente definir diretamente a proporção de famílias que conviviam com a situação de insuficiência de alimentos qualitativa e quantitativa. As informações geradas por esses indicadores creditavam à insegurança alimentar conceitos do baixo ní- 
vel econômico, da falta de acesso aos alimentos, do baixo consumo alimentar e da desnutrição, respectivamente 19 . Porém, apesar de os conceitos terem sido usados como sinônimos, a compreensão das diferenças é importante para uma avaliação criteriosa da dimensão da insegurança alimentar.

A pobreza não caracteriza a insegurança alimentar, pois a mesma se faz presente mesmo em países com alto desenvolvimento econômico. Da mesma forma, a insegurança alimentar se diferencia da baixa disponibilidade de alimentos, pois existem regiões com histórico sucessivo de altos índices de suprimento alimentar para consumo humano, todavia com adultos e crianças passando fome. O baixo consumo alimentar não deve ser confundido com insegurança alimentar, já que as carências nutricionais independem da situação fome, e podem estar presentes em lares com segurança alimentar. A última e mais importante miscigenação conceitual é derivada da vinculação entre insegurança alimentar e desnutrição. É sabido que a insegurança alimentar grave pode levar à desnutrição, por outro lado, as consequências de se viver em insegurança alimentar também passam pelo sobrepeso, quando há aumento no consumo de alimentos de alta densidade energética e baixo custo. Portanto, a EBIA, validada em 2004, passou a medir diretamente a experiência e a percepção de (in)segurança alimentar no domicílio como forma de dissociação de conceitos próximos porém distintos 8,11,19.

Utilizando dados da PNDS 2006, porém com um recorte dos domicílios brasileiros com crianças menores de cinco anos de idade e residentes com suas mães por ocasião do inquérito ( $\mathrm{n}=3.920$ ), evidenciou-se que são maiores as proporções nos diferentes graus de insegurança alimentar quando comparados com os dados da amostra total de domicílios pesquisados, para as macrorregiões do país. As prevalências de domicílios com moradores adultos e crianças que passavam fome na amostra total eram de $9,7 \%$ (IAM) e 4,8\% (IAG) 12 passando respectivamente, para $12,3 \%$ e $5,2 \%$ na subamostra de domicílios com crianças menores de cinco anos, representando incrementos proporcionais de $26,8 \%$ e $8,3 \%$. Portanto, as crianças menores de cinco anos, além de representarem um grupo de indivíduos mais suscetíveis à insegurança alimentar e nutricional devido ao processo de crescimento e desenvolvimento, também eram proporcionalmente mais acometidas pela falta de acesso aos alimentos em casa quando comparadas com a população total do país.

As estratégias familiares para enfrentar a insegurança alimentar iniciam-se quando os adultos responsáveis pela aquisição de alimen- tos, para poupar os comensais da sensação de fome, trocam qualidade por quantidade (IAL), uma vez que alimentos mais baratos tendem a ser mais calóricos, ricos em açúcares e/ou gorduras, induzindo à saciedade 20 . A opção por baixo preço para preservar a quantidade implica alimentação mais pobre em micronutrientes, e se por um lado, adultos e crianças encontram-se saciados com alimentos de alta densidade energética, por outro lado apresentam deficiências imunológicas e comportamentais devido à falta de micronutrientes e ao menor valor biológico das proteínas ingeridas 21 .

É importante destacar que há variações mais graves da insegurança alimentar, que dizem respeito à fome, que corrompem o Direito Humano à Alimentação Adequada (DHAA), ou seja, impugnam as condições necessárias e essenciais dos seres humanos desenvolverem suas capacidades, participando plenamente e dignamente da vida em sociedade 22. Quando à diminuição da qualidade da dieta, soma-se a falta de dinheiro para comprar alimentos em quantidade suficiente, acentua-se a condição de insegurança alimentar, situação na qual é habitual observar os adultos diminuírem a quantidade de alimentos visando à manutenção do consumo quantitativamente adequado para as crianças do domicílio (IAM). A prevalência de IAM nas macrorregiões Norte e Nordeste do país atingia um a cada cinco domicílios. Tais proporções quando expandidas para a população em seus estratos regionais representavam quase 900 mil domicílios, e residentes nestes, parentes de crianças menores de cinco anos vivendo em condição de incerteza quanto à disponibilidade financeira para alimentar a prole.

A segurança alimentar é condição necessária para o bem-estar físico, social e mental ${ }^{8}$. A impossibilidade de prover uma quantidade mínima de alimentos ameaça a confiança materna na sua habilidade de nutrir a prole, podendo causar ansiedade, melancolia, preocupação ou irritabilidade. Em adição, a falta de nutrientes diminui a sua própria energia afetando os cuidados e os estímulos de desenvolvimento físico e mental das crianças refletindo no vínculo mãe-filho 20,23. Por isso, a falta de qualidade dos cuidados recebidos, incluindo a nutrição, a assistência e o estímulo durante os primeiros anos de vida, pode ter um efeito negativo irreversível no desenvolvimento do cérebro, alterando a trajetória no processo de desenvolvimento do ser humano ${ }^{24}$. Assim, a depressão materna, como consequência ou causa da incerteza de poder alimentar a si e a seus filhos, pode ser um indicador indireto de que a insegurança alimentar reflete negativamente na saúde e no desenvolvimento das crianças 20,25. 
A situação mais grave da insegurança alimentar considera que além da diminuição da qualidade e da quantidade sentida pelos adultos, a falta de recurso financeiro da família impossibilita o acesso à quantidade de alimentos também para compor as refeições das crianças. Sendo assim, estes sentiram fome, seja por comer menos nas refeições, por pular refeições ou por passar dias inteiros sem se alimentar por falta de dinheiro, nos três meses que antecederam a pesquisa. A prevalência de IAG em domicílios brasileiros com crianças menores de cinco anos era cerca de um em cada 20 (5,2\%), mostrando que moradores adultos e crianças de mais de seiscentos mil domicílios passavam fome no país.

A insegurança alimentar é um problema de saúde pública no país, haja vista que no Brasil de 2006, pessoas residentes em quase metade dos domicílios brasileiros (45,5\%) viviam em algum grau de insegurança alimentar. As consequências de se viver assim são inúmeras. Para a saúde da criança estão associados à anemia 26,27 , à deficiência de vitamina A 28 e à ingestão inadequada de energia e proteína 21 .

Como viver em insegurança alimentar moderada e grave está associado a episódios de fome, é provável que adultos e crianças convivam com a inadequada ingestão de macro e micronutrientes. Programas de suplementação e fortificação de alimentos suprem apenas parte do problema. Em adição, o maior acesso a alimentos com alta densidade energético-proteica não resolve o problema, e ainda favorece excessos. Portanto, idealmente, seriam necessárias políticas de garantia do direito: “... ao acesso regular e permanente a alimentos de qualidade, em quantidade suficiente..." 22 .

Se foi estimado nesse estudo em um para 20 a proporção de IAG nos domicílios com crianças menores de cinco anos, dentre as classes menos favorecidas (D+E) a proporção de domicílios é duplicada, passando a ser de um para $10(10,8 \%)$. Pessoas menos favorecidas normalmente residem em ambientes precários e têm a nutrição comprometida, tornando-se, portanto, mais sujeitas à intercorrências de saúde. Em meio a tantas inseguranças higiênico-sanitárias, nutricionais e de saúde, estes cidadãos acabam por auferir rendimentos mais baixos, já que a produtividade de um indivíduo depende positivamente de suas condições de saúde e bem-estar. Portanto, crianças que crescem em domicílios menos favorecidos não apenas apresentam piores condições de saúde na infância, mas têm também sua capacidade produtiva reduzida no futuro, sendo adultos menos capazes de gerar renda e, por conseguinte, permanecendo na pobreza 29,30 . Sendo assim, a relação entre a condição de vida e saúde infantil, moldada pelo ambiente, pode representar importante fator na transmissão da desigualdade socioeconômica entre gerações.

Com o intuito de controlar a intergeracionalidade da desigualdade socioeconômica é que PTR são criados. Em 2004, o Bolsa Família ampliou a cobertura da população mais vulnerável à insegurança alimentar e, aos poucos, o governo federal aumentou, unificou e regularizou os benefícios sociais 31 .

Em meio a este processo, dentre a população que recebia algum benefício monetário governamental, quase um terço vivia em residências com adultos e crianças passando fome (IAM+G). Mas mesmo atingindo a população para a qual os PTR foram concebidos, o valor monetário repassado não é suficiente para retirar as famílias dos beneficiários da condição de insegurança alimentar, confirmando sua vulnerabilidade social em virtude da pobreza que é um fenômeno complexo e tem determinantes que a reproduzem permanentemente 32 .

O recebimento monetário aumenta o poder de compra de alimentos, mas não significa que os beneficiários estão optando por alimentos saudáveis ${ }^{33}$. Para ser efetivo, suscita-se a necessidade de programas paralelos de educação alimentar, principalmente para as mulheres, que são na sua maioria as titulares do benefício e também as gerenciadoras da alimentação e dos cuidados infantis 34. Em adição, alimentos saudáveis poderiam receber incentivos governamentais, como já é realidade a isenção de impostos federais, para os alimentos da cesta básica, hoje composta por carnes bovina, suína e ave, arroz, feijão, ovo, leite integral, café, açúcar, farinhas, pão, óleo, manteiga, frutas e legumes 35 . Concomitantemente, políticas públicas devem atentar para o outro lado do problema, conferindo especial atenção à iniciativa de sobretaxar alimentos industrializados que contenham excesso de nutrientes deletérios à saúde, como o sódio, as gorduras saturada e trans e o açúcar refinado. Iniciativa igualmente promissora é a normatização de mensagens de alerta sobre os riscos para a saúde do consumo continuado desses alimentos. Sendo assim, o consumidor seria esclarecido sobre os riscos do consumo de alimentos obesogênicos e estimulado financeiramente a autorregular o acesso a alimentos mais saudáveis, já que distúrbios nutricionais estão mais relacionados a dietas de baixa qualidade do que à privação de alimentos básicos 36 .

O modelo de análise multivariada (Tabela 2), ao identificar efeitos independentes de cada uma das variáveis associadas a $\mathrm{IAM}+\mathrm{G}$, corrobora a constatação dos riscos sociais (PTR), regionais (macrorregiões) e econômicos (ABEP) oferecen- 
do estimativas de quantificação de tais riscos para cada condição. O risco social (pertencer a PTR) é de 1,8, o regional (N+NE) é de 2,0 e o econômico (D+E) é da ordem de 2,4. Assim, cada uma dessas condições, de forma independente, duplica o risco de $\mathrm{IAM}+\mathrm{G}$. Estimativas similares de risco de $\mathrm{IAM}+\mathrm{G}$ foram identificadas em estudos pontuais no Brasil que também aplicaram o instrumento EBIA 37,38 .

Direitos Humanos são o conjunto de direitos universais, indivisíveis, inalienáveis, interdependentes e inter-relacionados, que os seres humanos possuem, por simplesmente terem nascido e serem da espécie humana 39 . DHAA é um direito humano que assegura o direito a se alimentar dignamente, de forma saudável e condizente com seus hábitos culturais 40 . O Estado é responsável por garantir os direitos da população e, dentre eles, o DHAA, por meio de políticas públicas que visam melhorar o acesso das pessoas aos recursos para produção ou aquisição, seleção e consumo de alimentos 41,42 .

A complexa realidade brasileira, geradora de desigualdades, exclusão social e pobreza viola o DHAA e necessita de urgente adoção de medidas corretivas eficazes. Apesar de os PTR atuarem na redução da desigualdade e na diminuição da miséria no país, a condição de estar em risco social, definida por participar desses programas, ainda confere aos domicílios prevalências $80 \%$ maiores $(\mathrm{RP}=1,8)$ de viverem em IAM+G quando comparadas com as residências onde não há pessoa pertencente a PTR.

Nesta mesma linha, a desigualdade cria bolsões macrorregionais onde são encontrados domicílios em situação econômica deficiente. Estar domiciliado nas macrorregiões $\mathrm{N}+\mathrm{NE}$ ou pertencer à classe social $\mathrm{D}+\mathrm{E}$ conferiram riscos de $100 \%$ e $140 \%$ maiores de estar em IAM+G, quando comparados com os domicílios das macrorregiões $\mathrm{CO}+\mathrm{SE}+\mathrm{S}$ ou pertencer às classes $\mathrm{A} 1$ à $\mathrm{C} 2$, respectivamente. Como o Bolsa Família, PTR de maior destaque em 2006, tinha o valor médio do benefício aquém do montante que permitiria à família sair da pobreza, o cenário das desigualdades econômicas regionalizadas pouco modificou-se 43 .

A análise das quatro situações de riscos socioeconômicos e demográficos, mostradas na Figura 1, considera a existência de vários Brasis diferentes quando aponta o aumento das proporções de $\mathrm{IAM}+\mathrm{G}$ na ocorrência simultânea de duas ou mais situações. Nos dois extremos identificou-se que a proporção de $\mathrm{IAM}+\mathrm{G}$ entre domicílios com um fator de risco (ser brasileiro e residir em domicílio com pelo menos uma criança menor de cinco anos) era de $17,5 \%$, representando mais de dois milhões de residências. Com os quatro fatores de risco agregados, esse valor quase triplicou (48\%), representando cerca de setecentas mil residências em situação de extrema vulnerabilidade.

O processo de identificação da população de maior risco para uma política social tem por objetivo tornar a distribuição dos recursos públicos específicos o mais eficiente possível, isto é, garantir que as transferências atendam, de fato, ao público-alvo pretendido 44 . Nesse processo, não é necessário criar novos programas, e sim estreitar e posteriormente expandir programas e equipamentos já existentes a fim de contemplar a população mais vulnerável, de diminuir a iniquidade e promover os direitos do cidadão, através da segurança alimentar 45 .

Incorporar estratégias de sustentabilidade fortalece as famílias residentes nos domicílios em insegurança alimentar para que possam responder positivamente à flutuação da acessibilidade ao alimento, sendo capazes de utilizar sua renda, ainda que complementada com PTR, para assegurar além da alimentação suas outras necessidades básicas como moradia, saúde e educação, ainda que essas famílias convivam com a vulnerabilidade de residir nas macrorregiões $\mathrm{N}+\mathrm{NE}$ e de pertencer às classes econômicas D+E. Para tanto, a EBIA deve ser utilizada contiguamente com indicadores e instrumentos de mensuração em distintas áreas aprofundando a compreensão deste fenômeno complexo que é a insegurança alimentar.

Além disso, é importante entender a natureza dinâmica da insegurança alimentar, ou seja, seu aspecto multifatorial inconstante, para que políticas públicas sejam capazes de planejar e implementar estratégias de intervenção consistentes com o desenvolvimento em longo prazo 34 . Entendendo-se que PTR são políticas paliativas e emergenciais que buscam minimizar o sofrimento humano enquanto não se concretizam mudanças de médio e longo prazos como são a educação de qualidade, a capacitação para o trabalho e a oferta de salário mínimo condizente com padrões aceitáveis de dignidade humana. 


\section{Resumen}

Se plantea estudiar el proceso de inseguridad alimentaria y el hambre en los hogares brasileños con niños menores de cinco años. Se trata de un estudio transversal a nivel nacional, realizado con datos de la Encuesta $\mathrm{Na}$ cional de Demografía y Salud de la Mujer y la Infancia (PNDS 2006), siendo la variable dependiente la inseguridad alimentaria moderada y grave $(I A M+G)$, medidas mediante la Escala Brasileña de Inseguridad Alimentaria (EBIA). Se generaron estimaciones de prevalencia y razón de prevalencia con intervalos de $95 \%$ de confian$z a$. Los resultados muestran una alta prevalencia de $I A M+G$ en el Norte y Nordeste (30,7\%), en las clases económicas D y E (34\%), y entre los beneficiarios de los programas de transferencias de renta (36,5\%). El modelo de análisis multivariado descubrió que los riesgos sociales (beneficiario de programas de transferencias de renta), regionales (Norte y Nordeste) y económicos (clases D y E) fueron de 1,8, 2, 0 y 2,4, respectivamente. Mediante la agregación de los tres riesgos se encontró el 48\% de los hogares en IAM+G, o sea, adultos y niños tuvieron hambre durante los tres meses anteriores a la encuesta.

Seguridad Alimentaria; Inequidad Social; Vulnerabilidad Social; Encuestas Epidemiológicas; Hambre

\section{Colaboradores}

A. P. Poblacion e J. A. A. C. Taddei contribuíram na concepção, projeto, análise e interpretação dos dados; redação do artigo e revisão crítica relevante do conteúdo intelectual; aprovação final da versão a ser publicada; são responsáveis por todos os aspectos do trabalho na garantia da exatidão e integridade de qualquer parte da obra. J. A. Silveira, L. Marín-León e A. M. Segall-Corrêa colaboraram na análise e interpretação dos dados; revisão crítica relevante do conteúdo intelectual; aprovação final da versão a ser publicada.

\section{Agradecimentos}

À Capes pela bolsa de doutoramento.

\section{Referências}

1. Programa das Nações Unidas para o Desenvolvimento. Sustentabilidade e equidade: um futuro melhor para todos. Estados Unidos, 2011. http:// hdr.undp.org/en/media/HDR_2011_PT_Comple te.pdf (acessado em 01/Mar/2013).

2. Neri MC. De volta ao país do futuro: crise europeia, projeções e a nova classe média. Rio de Janeiro: Fundação Getúlio Vargas; 2012.

3. Programa das Nações Unidas para o Desenvolvimento. Atlas do desenvolvimento humano no Brasil. Brasil, 2013. http://www.pnud.org.br/ IDH/Atlas2013.aspx?indiceAccordion=1\&li=li Atlas2013\# (acessado em 13/Set/2013).

4. Radimer KL, Olson CM, Campbell CC. Development of indicators to assess hunger. J Nutr 1990; 120 Suppl:1544-8.

5. Radimer KL, Olson CM, Greene JC, Campbell CC, Habicht J-P. Understanding hunger and developing indicators to assess it in women and children. J Nutr Educ 1992; 24 Suppl 1:36S-44.

6. Wehler CA, Scott RI, Anderson JJ. The Community Childhood Hunger Identification Project: a model of domestic hunger - demonstration project in Seattle, Washington, 1992. J Nutr Educ 1992; 24(1 Suppl 1):29S-35
7. National Research Council. Food insecurity and hunger in the United States: an assessment of the measure. Washington DC: National Academies Press; 2006.

8. Kepple AW, Segall-Corrêa AM. Conceituando e medindo a segurança alimentar e nutricional. Ciênc Saúde Coletiva 2011; 16:187-98.

9. Melgar-Quinonez H, Hackett M. Measuring household food security: the global experience. Rev Nutr 2008; 21 Suppl:27-37.

10. Segall-Corrêa AM, Pérez-Escamilla R, Sampaio MFA, Marin-Leon L, Panigassi G, Maranha LK, et al. Acompanhamento e avaliação da segurança alimentar de famílias brasileiras: validação de metodologia e de instrumento de coleta de informação. Urbano/Rural. Campinas: Universidade Estadual de Campinas; 2004. http://189.28.128.100/ nutricao/docs/geral/validacao_rural_urbano.pdf (acessado em 10/Dez/2012).

11. Segall-Corrêa AM, Marin-León L. A segurança alimentar no Brasil: proposições e usos da Escala Brasileira de Medida da Insegurança Alimentar (EBIA) de 2003 a 2009. Segurança Alimentar e Nutricional, Campinas 2009; 16:1-19. 
12. Ministério da Saúde. Pesquisa Nacional de Demografia e Saúde da Criança e da Mulher - PNDS 2006: dimensões do processo reprodutivo e da saúde da criança. Brasília: Ministério da Saúde; 2009.

13. Pérez-Escamilla R, Segall-Corrêa AM, Maranha LK, Sampaio MFA, Marin-León L, Panigassi G. An adapted version of the U.S. Department of Agriculture Food Insecurity Module is a valid tool for assessing household food insecurity in Campinas, Brazil. J Nutr 2004; 134:1923-8.

14. Marin-León L, Francisco PMSB, Segall-Corrêa AM, Panigassi G. Bens de consumo e insegurança alimentar: diferenças de gênero, cor de pele autorreferida e condição socioeconômica. Rev Bras Epidemiol 2011; 14:398-410.

15. Silva PLN, Pessoa DGC, Lila MF. Análise estatística de dados da PNAD: incorporando a estrutura do plano amostral. Ciênc Saúde Coletiva 2002; 7 : 659-70.

16. Berquó E, Garcia S, Lago T. Pesquisa Nacional de Demografia e Saúde da Criança e da Mulher - PNDS 2006. Aspectos metodológicos. http:// bvsms.saude.gov.br/bvs/pnds/img/Metodologia PNDS2006.pdf (acessado em 03/Ago/2013).

17. Rao J, Scott A. On chi-squared tests for multiway contingency tables with cell proportions estimated from survey data. Ann Stat 1984; 12:46-61.

18. Barros AJ, Hirakata VN. Alternatives for logistic regression in cross-sectional studies: an empirical comparison for models that directly estimate the prevalence ratio. BMC Med Res Methodol 2003; 3:21.

19. Kepple AW, Gubert MB, Segall-Corrêa AM. Instrumentos de avaliação de segurança alimentar e nutricional. In: Taddei JAAC, Lang RMF, LongoSilva G, Toloni MHA, organizadores. Nutrição em saúde pública. Rio de Janeiro: Editora Rubio; 2011. p. 77-93.

20. Cook JT, Frank DA. Food security, poverty, and human development in the United States. Ann NY Acad Sci 2008; 1136:193-209.

21. Cook JT, Frank DA, Berkowitz C, Black MM, Casey $\mathrm{PH}$, Cutts DB, et al. Food Insecurity is associated with adverse health outcomes among human infants and toddlers. J Nutr 2004; 134:1432-8.

22. Brasil. Lei ํo 11.346, de 15 de setembro de 2006. Lei Orgânica de Segurança Alimentar Nutricional. Cria o Sistema Nacional de Segurança Alimentar e Nutricional-SISAN com vistas em assegurar o direito humano à alimentação adequada e dá outras providências. Diário Oficial da União 2006; 18 set.

23. Whitaker RC, Phillips SM, Orzol SM. Food insecurity and the risks of depression and anxiety in mothers and behavior problems in their preschool-aged children. Pediatrics 2006; 118:e859-68.

24. Ramey CT, Ramey SL. Prevention of intellectual disabilities: early intervention to improve cognitive development. Prev Med 1998; 27:224-32.

25. Rose-Jacobs R, Black MM, Casey PH, Cook JT, Cutts DB, Chilton M, et al. Household food insecurity: associations with at-risk infant and toddler development. Pediatrics 2008; 121:65-72.
26. Park K, Kersey M, Geppert J, Story M, Cutts D, Himes JH. Household food insecurity is a risk factor for iron-deficiency anaemia in a multi-ethnic, low-income sample of infants and toddlers. Public Health Nutr 2009; 12:2120-38.

27. Skalicky A, Meyers AF, Adams WG, Yang Z, Cook JT, Frank DA. Child food insecurity and iron deficiency anemia in low-income infants and toddlers in the United States. Matern Child Health J 2006; 10:177-85

28. Oliveira JS, de Lira PI, Osório MM, Sequeira LA, Costa EC, Gonçalves FC, et al. Anemia, hypovitaminosis A and food insecurity in children of municipalities with Low Human Development Index in the Brazilian Northeast. Rev Bras Epidemiol 2010; 13:651-64.

29. Murphy C, Cuba SE, Cook JT, Cooper R, Weill JD. Reading, writing and hungry. The consequences of food insecurity on children, and our nation's economic success. Partnership for America's Economic Success 2008; (6). http://frac.org/newsite/ wp-content/uploads/2010/03/reading_writing_ hungry_report.pdf (acessado em 01/Jun/2013).

30. Reis M, Crespo A. O impacto da renda domiciliar sobre a saúde infantil no Brasil. Rio de Janeiro: Instituto de Pesquisa Econômica Aplicada; 2009. (Textos para Discussão, 1397).

31. Reis M. Food insecurity and the relationship between household income and children's health and nutrition in Brazil. Health Econ 2012; 21:405-27.

32. Segall-Corrêa AM, Marin-León L, Helito H, PérezEscamilla R, Santos LMP, Paes-Souza R. Transferência de renda e segurança alimentar no Brasil: análise dos dados nacionais. Rev Nutr 2008; 21 Suppl:39S-51.

33. Instituto Brasileiro de Análises Sociais e Econômicas. Repercussões do Programa Bolsa Família na segurança alimentar e nutricional. Relatório-síntese. Rio de Janeiro: Instituto Brasileiro de Análises Sociais e Econômicas; 2008.

34. Rose DD. Interventions to reduce household food insecurity: a synthesis of current concepts and approaches for Latin America. Rev Nutr 2008; 21 Suppl:159S-73.

35. Brasil. Medida Provisória no 609, de 8 de março de 2013. Reduz a zero as alíquotas da Contribuição para o PIS/PASEP, da COFINS, da Contribuição para o PIS/PASEP-Importação e da COFINS-Importação incidentes sobre a receita decorrente da venda no mercado interno e sobre a importação de produtos que compõem a cesta básica, e dá outras providências. Diário Oficial da União 2013; 8 mar.

36. Agência Nacional de Vigilância Sanitária. RDC nọ 24, de 15 de junho de 2010. Dispõe sobre a oferta, propaganda, publicidade, informação e outras práticas correlatas cujo objetivo seja a divulgação e a promoção comercial de alimentos considerados com quantidades elevadas de açúcar, de gordura saturada, de gordura trans, de sódio, e de bebidas com baixo teor nutricional, nos termos desta Resolução, e dá outras providências. Diário Oficial da União 2010; 29 jun. 
37. Vianna RPT, Segall-Corrêa AM. Insegurança alimentar das famílias residentes em municípios do interior de estado da Paraíba, Brasil. Rev Nutr 2008; 21:111-22.

38. Pimentel PG, Sichieri R, Salles-Costa R. Insegurança alimentar, condições socioeconômicas e indicadores antropométricos em crianças da região Metropolitana do Rio de Janeiro/Brasil. Rev Bras Estud Popul 2009; 26:283-94.

39. United Nations. The Universal Declaration of Human Rights, 1948. http://www.un.org/en/ documents/udhr/index.shtml (acessado em 05/ Ago/2013).

40. Comitê de Direitos Econômicos, Sociais e Culturais do Alto Comissariado de Direitos Humanos. O Direito Humano à alimentação (art.11). Comitê de Direitos Econômicos, Sociais e Culturais do Alto Comissariado de Direitos Humanos/ONU. http:// www.abrandh.org.br/download/20100702204835. pdf (acessado em 16/Fev/2013).

41. Pinheiro ARO, Carvalho MFCC. Transformando o problema da fome em questão alimentar e nutricional: uma crônica desigualdade social. Ciênc Saúde Coletiva 2010; 15:121-30.
42. Albuquerque MFM. A segurança alimentar e nutricional e o uso da abordagem de direitos humanos no desenho das políticas públicas para combater a fome e a pobreza. Rev Nutr 2009; 22:895-903.

43. Silva MOS. O Bolsa Família: problematizando questões centrais na política de transferência de renda no Brasil. Ciênc Saúde Coletiva 2007; 12:1429-39.

44. Tavares PA, Pazello ET, Fernandes R, Camelo RS. Uma avaliação do programa Bolsa Família: focalização e impacto na distribuição de renda e pobreza. Pesquisa e Planejamento Econômico 2009; 39:25-59.

45. Casey PH, Cuba SAE, Cook JT, Frank DA. Child hunger, food insecurity, and social policy. Arch Pediatr Adolesc Med 2010; 164:774-6.

Recebido em 26/Mar/2013

Versão final reapresentada em 01/Out/2013

Aprovado em 07/Nov/2013 